\title{
Improving the DiOlistics Imaging Technique: Visualizing Ventral Horn Spinal Motor Neurons in the Western Mosquitofish, Gambusia affinis affinis
}

\author{
Rodríguez-Cortes, A. ${ }^{1}$, B. N. Dugger ${ }^{2}$, N. L. Rivera-Rivera ${ }^{1}$, J. L. Serano-Velez ${ }^{1}$, and E. Rosa- \\ Molinar ${ }^{1}$. \\ ${ }^{1}$ University of Puerto Rico-Rio Piedras, San Juan, PR 00931-1809 and ${ }^{2}$ Michigan State University, \\ East Lansing, MI 48824-1044.
}

We describe modifications to the DiOlistics technique for rapid delivery, labeling, and visualization of ventral horn spinal motor neurons innervating the sexually dimorphic musculature of the anal fin, including their dendritic branching topology, dendritic varicosities, and fine spine morphology. Although a plethora of dyes have been available for neural tract tracing for 27 years, only recently has the gene gun been used for that purpose. The DiOlistics technique is similar to the ballistic transfer used by DNA vaccines and other gene bombardment with the gene gun, but it uses colloidal metal particles coated with dye instead of genetic material. As demonstrated in this study, the major advantage of the DiOlistics technique is that it makes it possible to conduct experiments in different parts of the nervous system of the same animal; the major disadvantages are limited tracing distances and long incubation times. However, despite some disadvantages, indocarbocyanine and oxacarbocyanines dyes are an indispensable tool for study of neuronal pathways.

References:

[1] Kiernan, J. A. Barr's The Human Nervous System An Anatomical Viewpoint, Lippincott Williams and Wilkins, Eighth Edition, 2004.

[2] Ramon y Cajal, S. Histology of the Nervous System of Man and Vertebrates. Translated from the French by N. Swanson and L. Swanson, 1995.

[3] Gan, W. B., et al. Multicolor "DiOlistic" Labeling of the Nervous System using Lipophilic Dye Combinations. Neuron 27(2):219-25, 2000.

[4] Wallace, W., et al. A Workingperson's Guide to Deconvolution in Light Microscopy. BioTechniques 31(5): 1075-1097 (2001).

[5] Rosa-Molinar, E. Proc. of the First International Symposium on Livebearing Fishes, New Life Publications, Inc., Homestead, Florida (book chapter in press) 2005.

[6] Research supported through NSF/IBN-0091120 and U54-NS39405-06 to ER-M.

Supported by NSF/IBN-0091120 and NIH/NS390405-06. 
Figure 1

A

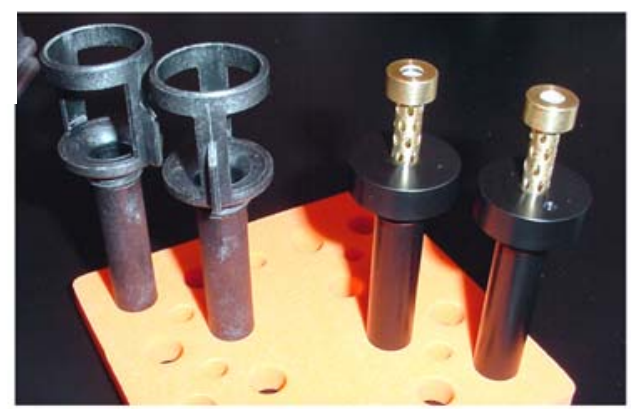

B

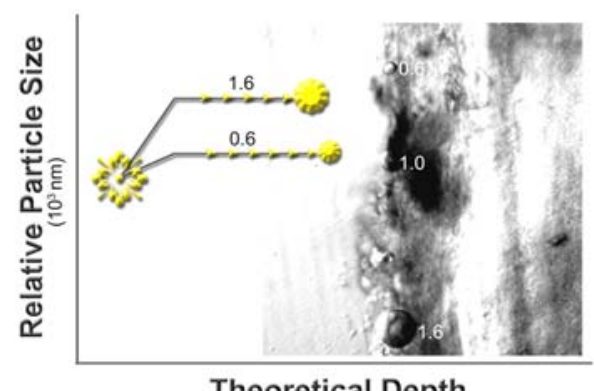

Theoretical Depth

Figure 1.

Helios Gene Gun and O'Brien barrels (A). DIC image of the spinal cord showing the penetration depth of the colloidal gold particles as a function of size (B).
Figure 2

A

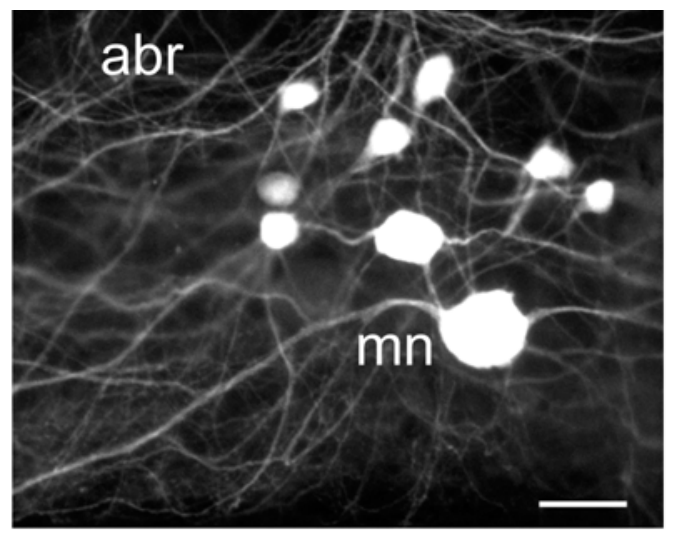

B

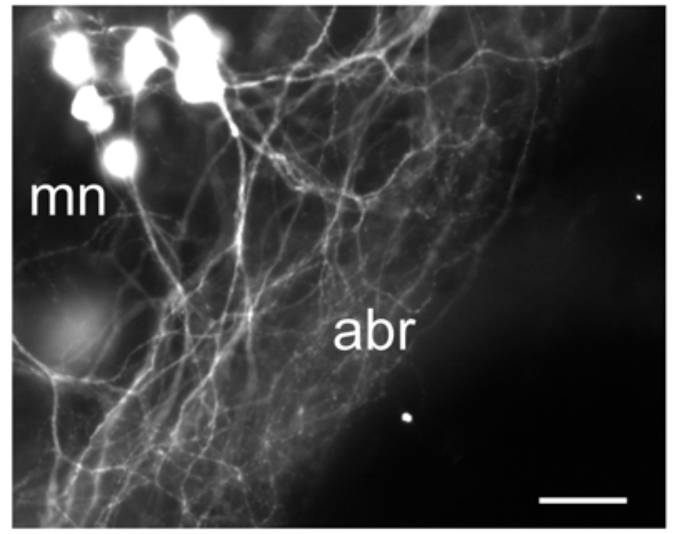

C

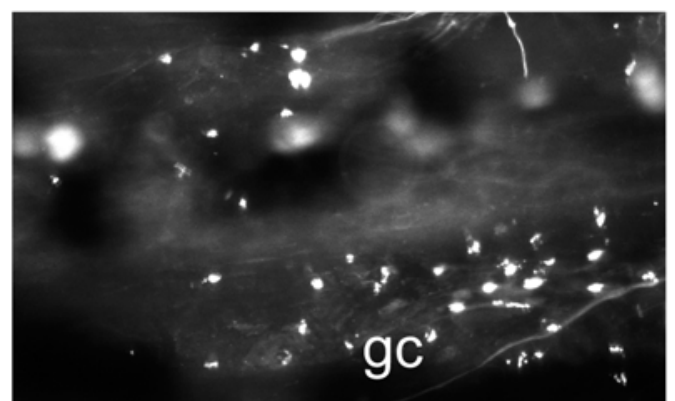

:

Figure 2.

DiOlistics label ventral horn spinal motor neurons [mn] (A-D). Note the extensive dendritic branching topology [abr] following application to the mEAM after 7 weeks incubation (A-B) Also note the colloidal gold clusters $[\mathrm{gc}]$ formed on the outside of the spinal cord (C) resulting in non-specific labeling of ventral horn spinal motor neurons (D).
D

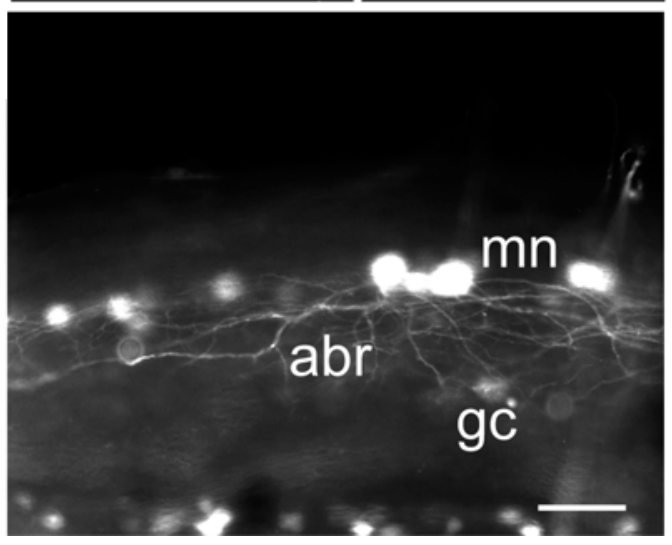

